\title{
GeoGebra en 2D y 3D como recurso didáctico en un curso de integración múltiple: una experiencia de enseñanza-aprendizaje
}

| GeoGebra in 2D and 3D as a learning resource in a multi-integration course: a teaching-learning experience |

\author{
Bolívar A. Ramírez Santamaría \\ bolivar.ramirez@ucr.ac.cr \\ Sección de Matemática \\ Universidad de Costa Rica \\ Costa Rica
}

Recibido: 16 junio 2019

Aceptado: 10 marzo 2020

\begin{abstract}
Resumen. El presente artículo tiene como finalidad exponer una experiencia de enseñanza-aprendizaje sobre el uso de GeoGebra, en dos y tres dimensiones, como un recurso didáctico para estudiar temas relacionados con integración múltiple. Para ello, se usó este software educativo en las clases y actividades de evaluación en un grupo del curso Principios de Análisis II, de la carrera de Bachillerato y Licenciatura de la Enseñanza de la Matemática, de la Sede Occidente de la Universidad de Costa Rica. Se utilizaron diferentes técnicas de recolección de información como exámenes parciales, cuestionarios y guías de observación. Se llega a la conclusión principal de que el uso de GeoGebra realmente promueve la motivación y la comprensión de los educandos en el estudio de temas sobre integración en varias variables.
\end{abstract}

Palabras clave: GeoGebra, enseñanza, aprendizaje, recurso didáctico, integración múltiple.

Abstract. The purpose of this research paper is to present a teaching and learning experience on the use of GeoGebra, in two and three dimensions, as a didactic resource used to study topics related to multiple integration. This educational software was used in classes and evaluation activities in a group of the course Principles of Analysis II, for the Bachillerato and Licenciatura in Mathematics Teaching of the University of Costa Rica, Sede de Occidente. Different information collection techniques were used such as partial exams, questionnaires and observation guides. The main conclusion is that GeoGebra promotes the motivation and understanding of the learners in the study of topics about to integration in several variables.

KeyWords: GeoGebra, teaching, learning, didactic resource, multiple integration.

\subsection{Introducción}

Históricamente es común que algunos estudiantes universitarios presenten ciertos problemas para comprender los conceptos matemáticos que involucran temas relacionados con el cálculo diferencial e integral de forma unidimensional, lo cual es evidenciado en diversas investigaciones educativas y es más notorio por los altos índices de reprobación (Artigué, 1995; Salinas, Alanís y Pulido, 2011; Parra y Díaz, 2014), más aún, "los alumnos universitarios deben atravesar grandes dificultades al pasar del estudio del Cálculo en una variable al estudio del Cálculo en dos o más variables" (Del Río, 2016, p.2).

Según lo anterior, en los cursos de la Educación Superior que involucran contenidos de cálculo en varias variables, pueden existir importantes complicaciones en el aprendizaje de integración en dos y tres variables. Estos problemas, en gran medida, obedecen a la dificultad de los educandos de realizar gráficas, tanto en dos como en tres dimensiones, solo utilizando lápiz y papel (Del Río, 2016). Esto, a su vez, obliga al estudiante a limitar la manipulación algebraica, repercutiendo negativamente en el razonamiento matemático sobre los temas estudiados en la clase. 
En este sentido, si el discente no logra una correcta interpretación gráfica de cierta región en dos o tres dimensiones para plantear una integral doble o triple, es probable que la resolución de éstas sea incorrecta. Dicha apreciación la afirman Vicente, Trefftz y Restrepo (2006), al escribir “... las experiencias de aprendizaje en las que intervengan representaciones visuales asociadas a los conceptos matemáticos son necesarias para integrarlos con aplicaciones relacionadas ..." (p.121).

Además, los mismos conflictos cognoscitivos descritos anteriormente se presentan en las integrales de línea e integrales de superficie, ya que su resolución está profundamente ligada a las integrales dobles y triples, sobre todo si se usan ciertos teoremas como el Teorema de Green, el Teorema de la Divergencia de Gauss y el Teorema de Stokes. Entonces se genera una tensión entre la necesidad de manipular y articular algebraicamente y la dificultad agregada que reviste el uso de la interpretación de gráficos bidimensionales y tridimensionales, usando solo lápiz y papel.

Sumado a lo anterior, Andrade y Montecino (2011), por medio de una investigación, concluyeron que los estudiantes presentan muchas dificultades cuando se deben realizar representaciones tridimensionales, y aún más cuando deben acudir a ella para encontrar la solución a cierto problema.

Expuesta la problemática, y de acuerdo con Gómez y Delgado (2012), existe una “...necesidad de cambiar el tratamiento metodológico que tradicionalmente se hace en los cursos de Cálculo de varias variables ..." (p.2). Al respecto, este artículo tiene como finalidad reflexionar sobre el uso de GeoGebra como un recurso didáctico para representar y visualizar las regiones en dos y tres dimensiones, esto en un curso de integración múltiple, bajo la hipótesis de que esto puede promover la comprensión de los temas estudiados.

\subsection{Antecedentes}

De acuerdo con Trigueros y Martínez-Planell (2010) y Dorko y Weber (2014), existen pocos estudios acerca de cómo los estudiantes desarrollan su comprensión sobre las funciones en varias variables. Sin embargo, Trigueros y Martínez-Planell (2010) hacen referencia a que entender las propiedades geométricas y algebraicas de las funciones estás relacionadas con la visualización.

Además, según dicha investigación, hay diferencias importantes en la capacidad para graficar funciones de dos variables y para obtener una noción intuitiva de las gráficas de tres dimensiones, más cuando se presentan intersecciones entre superficies y se deben obtener proyecciones. Los autores concluyeron que la comprensión de las gráficas de funciones de dos variables no es fácil para los estudiantes, siendo la principal causa la incapacidad de imaginar cómo se genera la gráfica por parte de los educandos.

Por su lado, Andrade y Montecino (2011), escriben que "La visualización espacial puede utilizarse como recurso pedagógico (por parte del profesor) o herramienta (por parte del alumno) para facilitar la comprensión y el entendimiento de saberes matemáticos abstractos involucrados en el proceso de transposición o en la interiorización de éstos por parte de los estudiantes" (p.2). En el estudio de Andrade y Montecino (2011), en sus conclusiones insisten en realizar nuevas estrategias de enseñanzaaprendizaje, que beneficien las compresiones matemáticas, especialmente lo referente a lo geométrico, de forma dinámica y variable, apoyándose de ciertos recursos tecnológicos.

Igualmente, en la investigación realizada por Weber y Thompson (2014), permiten a los educandos utilizar una Calculadora Gráfica para crear gráficas en dos y tres dimensiones. Este instrumento se utilizó sobre todo para confirmar o no lo que creían estar visualizando. Al respecto, los investigadores consideran que la capacidad de confirmar las ideas utilizando la Calculadora Gráfica les permitió tener mayor seguridad a los alumnos a la hora de resolver ejercicios. Estos investigadores recalcan la importancia de entender el proceso cognitivo de los estudiantes cuando generan e interpretan gráfi- 
cas de funciones de más de una variable.

Otro estudio relevante es el realizado por Vieira (2012), donde se destaca el papel de la tecnología para promover la visualización y la aprehensión perceptiva de propiedades de objetos matemáticos en el espacio. En la investigación de Vieira (2012), se implementan GeoGebra y CAS Maple para visualizar gráficas como apoyo para mejorar la comprensión de temas sobre el cálculo diferencial en dos variables.

Por su parte, Mora (2019), ha desarrollado un texto interactivo para un curso de cálculo en varias variables, donde el lector puede interactuar con las figuras, empleando el ratón. El objetivo es visualizar el espacio tridimensional, enfocándose en los cortes de superficies, las intersecciones y las proyecciones de una superficie o un sólido. En el documento realizado por Mora (2019), algunas aplicaciones se utilizan para visualizar la dinámica de una definición o un teorema, recalcando en que la visualización interactiva funciona como un complemento y requiere el apoyo por parte del profesor para obtener mejores resultados en el proceso de enseñanza-aprendizaje.

\subsection{Las herramientas tecnológicas en la formación matemática}

En las últimas décadas el desarrollo tecnológico se ha convertido en un eje fundamental en el progreso social y educativo. Bajo este indicio, la educación terciaria debe adaptarse a estos cambios para optimizar el uso de las Tecnologías de la Información y la Comunicación ( $\mathrm{TIC}^{1}$ ) como un valioso recurso didáctico para el desarrollo de las clases. En relación con esto, Parra y Díaz (2014) establecen que "En la educación y específicamente en las matemáticas el impacto de las Tecnologías de la Información y Comunicación ha revolucionado la forma de enseñar y orientar el conocimiento e interacción formativa que se presenta en todos los niveles de la educación en matemáticas" (p.75).

De hecho, el panorama de la tecnología está cambiando con rapidez; hoy en día se cuentan con dispositivos móviles como tabletas y teléfonos inteligentes de gran gama y con interfaces táctiles, los cuales tienen muchas de las funciones de un ordenador de escritorio. En particular, existe una enorme cantidad de aplicaciones que permiten a los usuarios explorar las Matemáticas, a dar sentido a los conceptos y procedimientos, permitiendo un aprendizaje más significativo al favorecer el razonamiento matemático (National Council of Teachers of Mathematics, 2015, p.78). Abonado a ello, Parra y Díaz (2014) escriben que

... al incidir en procesos complejos de visualización y representación se lleva al estudiante a fortalecer sus esquemas intuitivos, creando en él un desarrollo de reconocimiento y aprehensión de los fenómenos descritos para su contextualización, en veras de una descripción y exploración a partir de las herramientas que ofrece hoy día la tecnología y sus condiciones en el ámbito de las matemáticas (p.77).

Inclusive, el uso de la tecnología da cabida a la realización de actividades distintas e innovadoras para el proceso de enseñanza-aprendizaje en Matemáticas, y a su vez, permite al discente desarrollar nuevas experiencias de formación. Más aún, las TIC se han convertido gradualmente en un agente transformador del proceso de cambio en la Educación Matemática, ya que “... abre espacios para que el estudiante pueda vivir nuevas experiencias matemáticas (difíciles de lograr en medios tradicionales como el lápiz y el papel) en las que él puede manipular directamente los objetos matemáticos dentro de un ambiente de exploración" (Gómez, 1997, p.93).

A pesar de estas bondades de las TIC, es importante aclarar que no son la solución definitiva a los problemas de la enseñanza-aprendizaje de las Matemáticas (Gómez, 1997). Sin embargo, “El principal

\footnotetext{
${ }^{1}$ En algunos escritos se lee Tecnologías Digitales, abreviado como TD.
} 
aporte de la tecnología consiste en que la interacción entre ella, el profesor y el estudiante, está cambiando la visión que los actores tienen del contenido matemático y del proceso didáctico" (Infante, Quintero y Logreira, 2010, p.37).

Por ello, en la época actual, es indispensable que la Educación Matemática en las universidades (y en todo ámbito de la escolarización) no se enajene del uso de las TIC, porque son una herramienta didáctica muy poderosa y para la cual se necesita de docentes proactivos y reformadores que den un uso adecuado de dicho recurso, recalcando que "El profesor es quien tiene la responsabilidad de diseñar las situaciones didácticas más apropiadas para aprovechar las potencialidades de la tecnología de acuerdo a las dificultades y las necesidades de los estudiantes" (Gómez, 1997, p.106).

En resumen, bajo una planificación acertada por parte del docente, cualquier estudiante puede beneficiarse del uso de materiales virtuales que se puedan manipular, esto con el fin de proporcionar modelos visuales para respaldar el razonamiento matemático y la capacidad para la resolución de problemas (National Council of Teachers of Mathematics, 2015).

Basándose en lo anterior, y recapitulando el problema de aprendizaje que presentan los educandos en temas de integración múltiple, esto debido a la dificultad que presentan al realizar gráficas tanto en dos como en tres dimensiones, únicamente utilizando medios tradicionales como el lápiz y el papel; la tecnología merece ser un valioso aliado pedagógico que pueda ayudar significativamente a los estudiantes a superar este tipo de obstáculos.

Más aún, al incorporar las nuevas tecnologías en la enseñanza para permitir otros tipos de aprendizaje, las cuales a su vez permiten introducir imágenes y animaciones, pueden motivar a los alumnos (Costa, Di Domenicantonio y Vacchino, 2010).

Bajo estas premisas, Vicente et al. (2006) mencionan “... con los avances en computación, es posible diseñar experiencias de aprendizaje significativas que permitan comprender los conceptos matemáticos con ayuda de la visualización" (p.122). En este sentido,

... en la actualidad, existen recursos informáticos que permiten graficar con facilidad, que pueden ser útiles para promover el uso por parte del alumno del registro gráfico. Además, este tipo de recursos posibilita representaciones de los objetos tridimensionales más ricas que las planas, que pueden hacerse en soporte impreso, ya que puede rotarse el punto de vista con facilidad... (Del Río, 2016, p.3).

Más enfocado al tema de estudio del presente artículo, se resalta que Vieira (2012) refuerza las ideas anteriores, pues considera que con un fuerte énfasis en la visualización de los objetos matemáticos por medio de alguna herramienta tecnológica, contribuye en la transición que debe realizar el educando cuando pasa de estudiar cálculo en una variable al cálculo en varias variables.

Por tanto, en aras de beneficiar el aprendizaje de temas relacionados con integrales en dos y tres variables, una herramienta informática que puede ser de gran utilidad didáctica es GeoGebra.

\subsection{Un poco sobre el software GeoGebra}

Según la página oficial² de GeoGebra, se establece que

GeoGebra es un software de matemáticas dinámicas para todos los niveles educativos que reúne geometría, álgebra, hoja de cálculo, gráficos, estadística y cálculo en un solo programa fácil de usar. GeoGebra es también una comunidad en rápida expansión, con millones de usuarios en casi todos

\footnotetext{
${ }^{2}$ https://www.geogebra.org/
} 
los países. GeoGebra se ha convertido en el proveedor líder de software de matemática dinámica, apoyando la educación en ciencias, tecnología, ingeniería y matemáticas (STEM: Science Technology Engineering and Mathematics) y la innovación en la enseñanza y el aprendizaje en todo el mundo.

Se recalca que dicho software es de uso libre y está disponible en diversas plataformas: macOS, Windows, Linux, Android e iOS. También, en la actualidad, existe una comunidad de docentes, investigadores, estudiantes y otras personas interesadas en el uso de GeoGebra, las cuales trabajan ya sea de forma individual o grupal. Inclusive existen diferentes Institutos GeoGebra locales que articulan entre sí por medio del Instituto GeoGebra Internacional ${ }^{3}$.

En este sentido, es notorio que GeoGebra es un valioso recurso para la Educación Matemática porque permite diseñar, construir, explorar y resolver problemas de manera interactiva (Carrillo y Llamas, 2009), favoreciendo la motivación del educando. Más aún, sobre esta tecnología digital, Santana y Climent (2015) mencionan que “... el conocimiento de cómo aprenden los estudiantes determinados contenidos matemáticos y algunas de las dificultades habituales (como en nuestro caso la dificultad para pensar en general, sin visualizaciones concretas) puede motivar la necesidad de la herramienta y contribuir a apreciar sus ventajas" (p.90).

Además, su vista gráfica en tres dimensiones fue incorporada recientemente. Por tanto, se debe trabajar en propuestas que permitan explotar las nuevas posibilidades que brinda para la enseñanza y el aprendizaje (Del Río, 2016), lo cual es parte de lo que se describe en el presente trabajo.

\subsection{Aspectos Metodológicos}

La investigación consistió en utilizar el software GeoGebra en un curso de Matemática donde se evalúe contenidos sobre integración en varias variables, bajo la hipótesis de que dicho uso puede beneficiar la enseñanza y el aprendizaje de los temas estudiados.

Así, la investigación es cualitativa, ya que es una actividad sistemática orientada a la descripción y comprensión de un fenómeno educativo (Bisquerra, 2009), siendo éste el análisis y la reflexión a partir de la implementación de GeoGebra como un recurso didáctico en la resolución de ejercicios de integración en varias variables.

Con este fin, se trabajó con un grupo del curso Principios de Análisis II, sigla MA0551, de la Carrera de Bachillerato y Licenciatura en la Enseñanza de la Matemática ${ }^{4}$, esto en la Universidad de Costa Rica, Sede Occidente, durante el segundo ciclo semestral del año 2017. Este curso es de cuarto año de carrera, de 5 horas lectivas semanales repartidas en 2 días y 7 horas de trabajo independiente del estudiante. Se estudian contenidos que versan sobre:

- Integración de dos y tres variables.

- Integrales de línea.

- Integrales de superficie.

- Teorema de Green, Teorema de Stokes y Teorema de la Divergencia de Gauss.

El curso usualmente se aborda de manera formal y práctica, dando énfasis en el planteo y cálculo de integrales (dobles, triples, de línea y de superficie), cuya bibliografía más usada corresponde a Pita (1995), Stewart (2002) y Apostol (2004). Sin embargo, y de acuerdo con los programas de los

\footnotetext{
${ }^{3}$ En Costa Rica, el Instituto GeoGebra se ubica dentro del Tecnológico de Costa Rica (TEC), en Cartago.

${ }^{4}$ Se aclara que los grupos de cuarto año de la Carrera de Bachillerato y Licenciatura en la Enseñanza de la Matemática, son grupo pequeños, que no suelen sobrepasar los 10 estudiantes.
} 
útimos cinco años del curso MA0551, no se han utilizado herramientas tecnológicas que favorezcan un aprendizaje significativo. De esta manera, a continuación se describen brevemente las actividades que se desarrollaron en clases para validar la hipótesis de investigación:

- Cada estudiante debía traer a todas las clases su computadora portátil, tableta o teléfono inteligente con GeoGebra instalado. El docente contaba con una aula de dos pizarras acrílicas, un video proyector y una pantalla de proyección.

- En la primera semana de clases se hizo una explicación general sobre el uso de GeoGebra, sobre todo de cómo graficar curvas, en dos dimensiones y tres dimensiones, y superficies, ya sean parametrizadas o no. Se debe considerar que los educandos, durante su tercer año de carrera, aprobaron un curso de Geometría Analítica, por tanto, los aspectos algebraicos en relación con curvas y superficies parametrizadas son conocimientos que se asumen adquiridos, además de las gráficas de éstas.

- Las clases fueron semi-magistrales, en el sentido que el profesor explicaba la teoría y daba ejemplos, en cuya solución, en la mayoría de los casos, se acudía al uso de GeoGebra. De igual manera, los educandos debían resolver ejercicios, interpretando la resolución por medio de los dibujos hechos en GeoGebra, para posteriormente revisar las respuestas en conjunto.

- Para las pruebas parciales de evaluación del curso, el estudiante podía recurrir al uso de GeoGebra, teniendo claro que es una herramienta que brindará soporte en la resolución y el razonamiento.

- Finalmente, por medio de la observación participante, un cuestionario para los estudiantes y las tres pruebas parciales del curso (en las cuales debían usar GeoGebra para dar solución a diversas interrogantes), se reflexionó sobre los resultados obtenidos. Se destaca que las técnicas e instrumentos para la recolección de información obedecen a una investigación cualitativa (Barrantes, 2014). En particular, el cuestionario tenía preguntas abiertas y cerradas. Para estas últimas, se usó una escala del 1 al 5 para apreciar el grado de satisfacción sobre el uso de GeoGebra en los diferentes temas, donde también podían opinar sobre el número seleccionado en dicha escala. Con ello, se pretendía conocer la opinión de los discentes sobre el uso del software susodicho, tanto en las actividades cotidianas de las clases como en las actividades de evaluación.

\subsection{Análisis empírico del uso de GeoGebra como recurso didáctico}

Inicialmente se realiza un análisis de los resultados de las tres pruebas parciales del curso, donde el uso de GeoGebra fue permitido, teniendo claro que el empleo de este paquete computacional no era el fin, sino el medio para construir, manipular e interpretar modelos visuales en dos y tres dimensiones, y así beneficiar el razonamiento matemático y la capacidad para la resolución de problemas. Posteriormente, se realiza un análisis de los datos obtenidos a partir de la observación participante y el cuestionario.

Análisis de los resultados de la primera prueba parcial.

El primer examen parcial constaba de cinco preguntas de desarrollo con un valor total de 100 puntos, de los cuales, 28 puntos correspondían a preguntas formales sobre el tema de integración en dos variables, cuyo uso de GeoGeebra no es viable. Los otros 72 puntos correspondían a planteo y resolución de integrales dobles, considerando cambios de variables y su aplicación en áreas de regiones en el plano y en volúmenes de regiones en el espacio, donde podían apoyarse en GeoGebra para darle resolución. A continuación se aprecian algunas de estas preguntas.

- Plantee, tanto en el orden $d y d x$ como $d x d y$, la integral doble que permita calcular el área de la región limitada por las curvas $x^{2}+y^{2}=4, x^{2}+y^{2}=4 x, x+y=4$ y y $=0$. 
Para esta pregunta el uso de GeoGebra les benefició para interpretar de forma más rápida y segura los límites de integración, además de las intersecciones entre las figuras involucradas. De esa forma les permitió enfocarse más en el planteamiento de la integral doble solicitada. La figura 1.6 muestra lo que hizo un alumno con el software, donde se observa sombreada la región de integración.

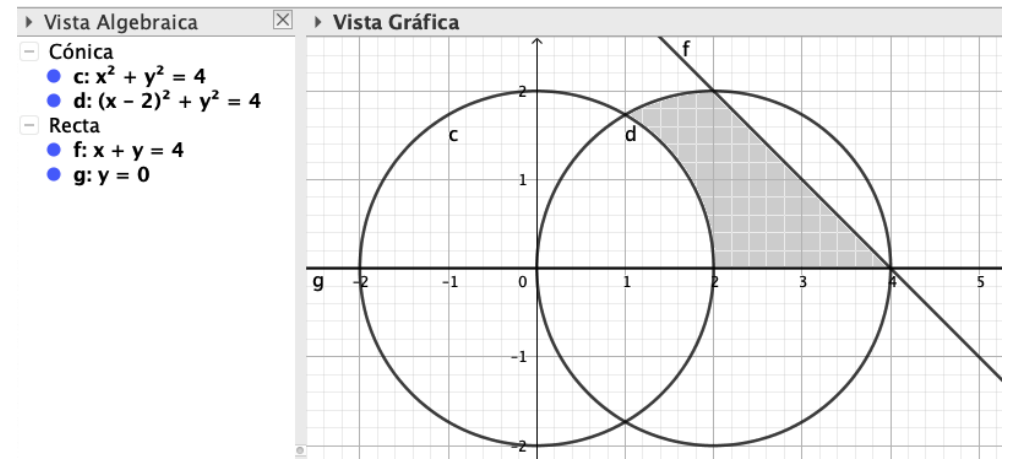

Figura 1.1: Región dibujada por un estudiante en GeoGebra y remozada por el docente.

La intersección entre las dos circunferencias en el primer cuadrante fácilmente se observa que ocurre cuando $x=1$ y esto se sustituye en cualquiera de las dos curvas, obteniendo $y=\sqrt{3}$. Sin embargo, algunos pocos estudiantes determinaron el valor de $x$ y $y$ hallando algebraicamente la intersección entre las dos circunferencias, que aunque sigue siendo un proceso sencillo, no era el objetivo, ya que GeoGebra proporciona visualmente el valor de $x$ y a partir de ello obtener el valor de y, como se hizo al inicio de este párrafo.

Posteriormente, el apoyo de GeoGebra se evidenció aún más en el proceso para describir cada subregión involucrada según el orden de integración, ya que al poder agrandar el dibujo y manipularlo a conveniencia, les facilitó la visualización, y por ende les ayudó a plantear lo solicitado. En la figura 1.6 se aprecia el dibujo hecho por medio de GeoGebra de un estudiante para ambos órdenes de integración.

El error más significativo cometido por un educando fue despejar de forma incorrecta la variable $x$ de la ecuación $x^{2}+y^{2}=4 x$, pero la interpretación de las subregiones de acuerdo con el orden de integración fue correcto tanto para este alumno como para todos los demás, haciendo que el planteamiento de las integrables dobles no sea completamente equivocado.
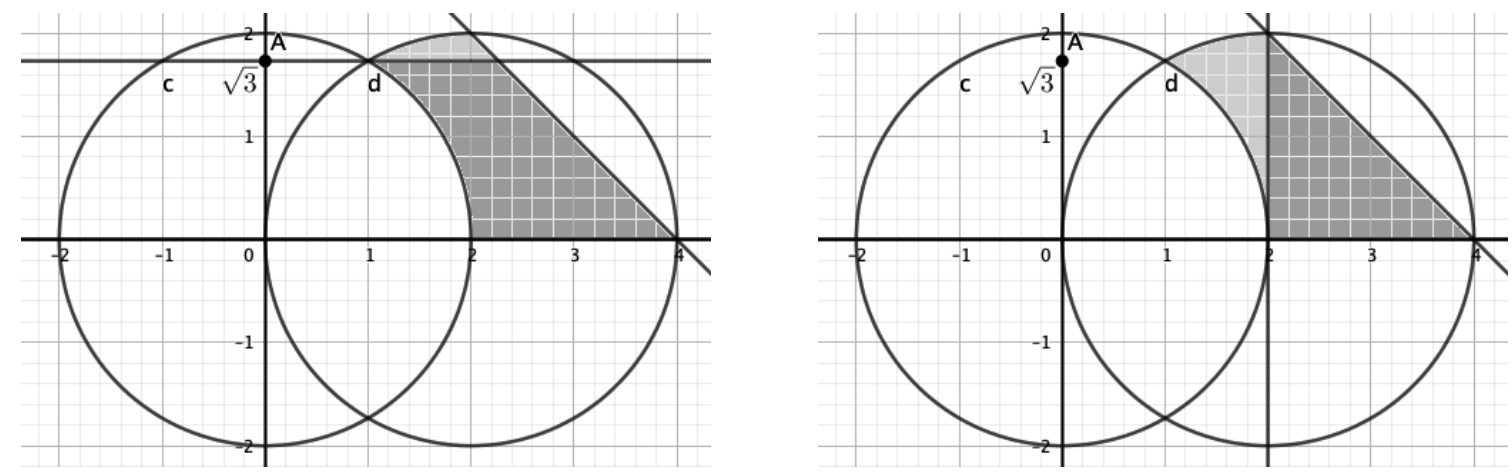

Figura 1.2: Región en el orden $d x d y$ y dydx dibujada por un estudiante en GeoGebra y remozada por el docente.

- Sea $R$ la región encerrada por las cuatro rectas $y=-x+\frac{1}{2}, y=-x+1, y=x-\frac{1}{2}$ y $y=x-1$. Sea la integral

$$
I=\iint_{R} \frac{1}{\sqrt{x^{2}-y^{2}}} d A .
$$


Calcule el valor de I usando el cambio de variables $x=(u+1)^{2}+(v+1)^{2}$ y $y=(u+1)^{2}-(v+1)^{2}$, donde $u>-1$ y $v>-1$. Además, dibuje $R$ y $R^{\prime}$.

Igual que la pregunta anterior, los estudiantes rápidamente dibujaron la región $R$, visualizando la frontera e identificando de forma fiable las intersecciones involucradas, con la cual debieron trabajar para el cambio de variable sugerido. Después de un análisis correcto de la frontera de $R$, se llega a las rectas que delimitan $R^{\prime}$ y se dibuja fácilmente por medio de GeoGebra. En este sentido, el apoyo del software fue para visualizar las regiones $R$ y $R^{\prime}$ y a partir de esto apoyarse en el planteamiento de la integral doble.

Los errores cometidos siguen siendo de naturaleza algebraica, sobre todo en el cálculo de la integral doble, sin embargo, el planteamiento de ésta en cuanto a los límites de integración fue correcta en casi todos los alumnos.

- Usando integrales dobles y coordenadas polares, determine el volumen del "cono de helado", limitado superiormente por la semiesfera $x^{2}+y^{2}+z^{2}=1, z \geq 0$, e inferiormente por el semicono $x^{2}+y^{2}-z^{2}=0, z \geq 0$.

Esta fue la interrogante que más fallaron, pero similarmente que las anteriores, obedeció a malos cálculos para hallar el valor de la integral doble en coordenadas polares, la cual fue planteada de forma correcta por la mayoría de estudiantes.

El beneficio de utilizar GeoGebra se constató en el dibujo del sólido, y más aún en la manipulación y análisis de éste, en el sentido que los educandos lo rotaron para visualizar eficazmente las intersecciones involucradas y la proyección en el plano xy, lo cual es de gran ayuda para plantear la integral doble en coordenadas polares que permita calcular el volumen. La figura 1.6 presenta un boceto elaborado por un alumno, donde se observa el sólido en primera instancia y usando la herramienta de rotación, en segunda instancia se aprecia la proyección del sólido en el plano xy.
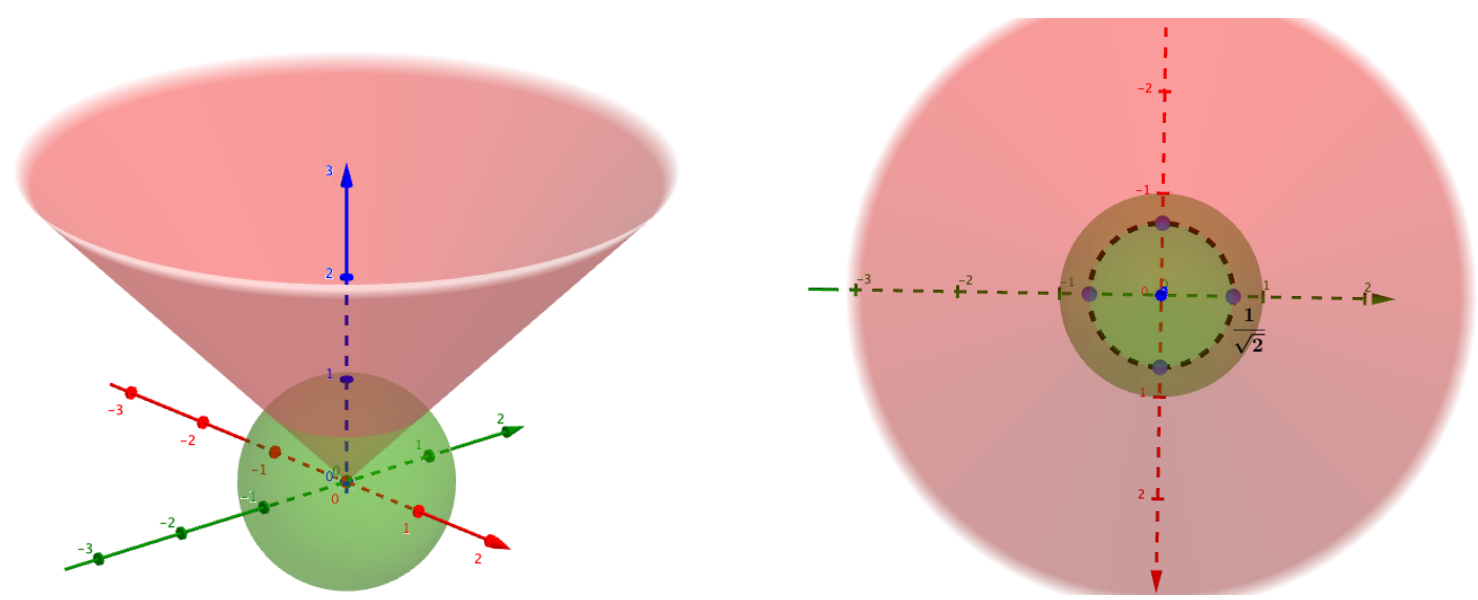

Figura 1.3: El sólido y su proyección en el plano xy elaborada por un estudiante en GoeGebra 3D.

En general y en síntesis, de los 72 puntos de las interrogantes del primer examen parcial y en las cuales los educandos podían apoyarse en GeoGebra, la suma ponderada obtenida entre todos los estudiantes fue de 55 puntos, es decir, obtuvieron un 76,4\% de rendimiento, lo cual es aceptable.

Análisis de los resultados de la segunda prueba parcial. La segunda prueba parcial también constaba de cinco preguntas de desarrollo, con un valor total de 100 puntos, de los cuales, 30 puntos correspondían a interrogantes sobre el tema de Campos Conservativos, donde el uso de GeoGebra 
no era de utilidad. Los otros 70 puntos fueron sobre planteo y resolución de integrales triples, considerando cambios de variables y su aplicación en volúmenes de regiones en el espacio, donde los estudiantes podían recurrir a GeoGebra para beneficiar el razonamiento matemático con el fin de resolver las interrogantes.

Para este examen parcial se notó un aspecto muy interesante e importante de rescatar: los estudiantes cada vez realizaban dibujos más elaborados por medio de GeoGebra en tres dimensiones. Esto se debió a que conforme avanzaba el curso y se usaba más dicho paquete computacional, se fueron conociendo más herramientas de mucha utilidad dentro este software, tales como intersección entre sólidos, graficar curvas y superficies parametrizadas en el espacio, modificar los aspectos de las superficies, entre otros. Así, los alumnos adquirieron una mayor destreza con el uso de GeoGebra en tres dimensiones. A continuación se presentan algunas de las interrogantes donde se podía usar este software educativo.

- Sea $\mathrm{Q}$ el sólido ubicado en el primer octante y limitado por los planos $x+z=4$ y y $+z=4$. Considere la integral $I=\iiint_{Q} f(x, y, z) d V$.

a. Escriba I en el orden $d x d z d y$.

b. Escriba I en el orden $d y d z d x$.

c. Escriba I en el orden $d z d y d x$.

Posiblemente esta sea una de las preguntas donde mayor beneficio obtuvieron los educandos con el uso de GeoGebra. La elaboración del sólido Q lo hicieron graficando las superficies involucradas para luego visualizar las intersecciones, y usando otras herramientas que proporciona el software detallaron los límites del sólido.

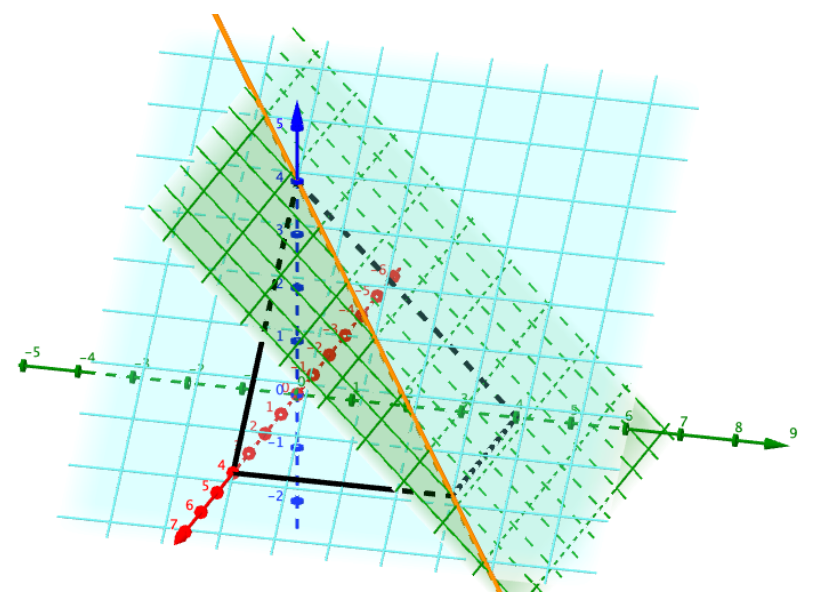

Figura 1.4: Visualización del sólido elaborada por una estudiante en GeoGebra 3D.

En la figura 1.6 anterior se aprecia un bosquejo del sólido Q hecho por una estudiante, cuyo diseño fue más elaborado. Esto obedece a que los alumnos han adquirido mayores destrezas con el uso de GeoGebra, según se explicó con anterioridad. Posteriormente, y con la finalidad de escribir la integral triple en los órdenes de integración solicitados, con la herramienta de rotación que ofrece GeoGebra en 3D, los alumnos visualizaron las proyecciones del sólido Q en los diferentes planos. La figura 1.6 muestra el sólido Q hecho por la misma estudiante, pero rotado de tal forma que se observen las proyecciones en los planos $y z, x z y x y$, respectivamente. 

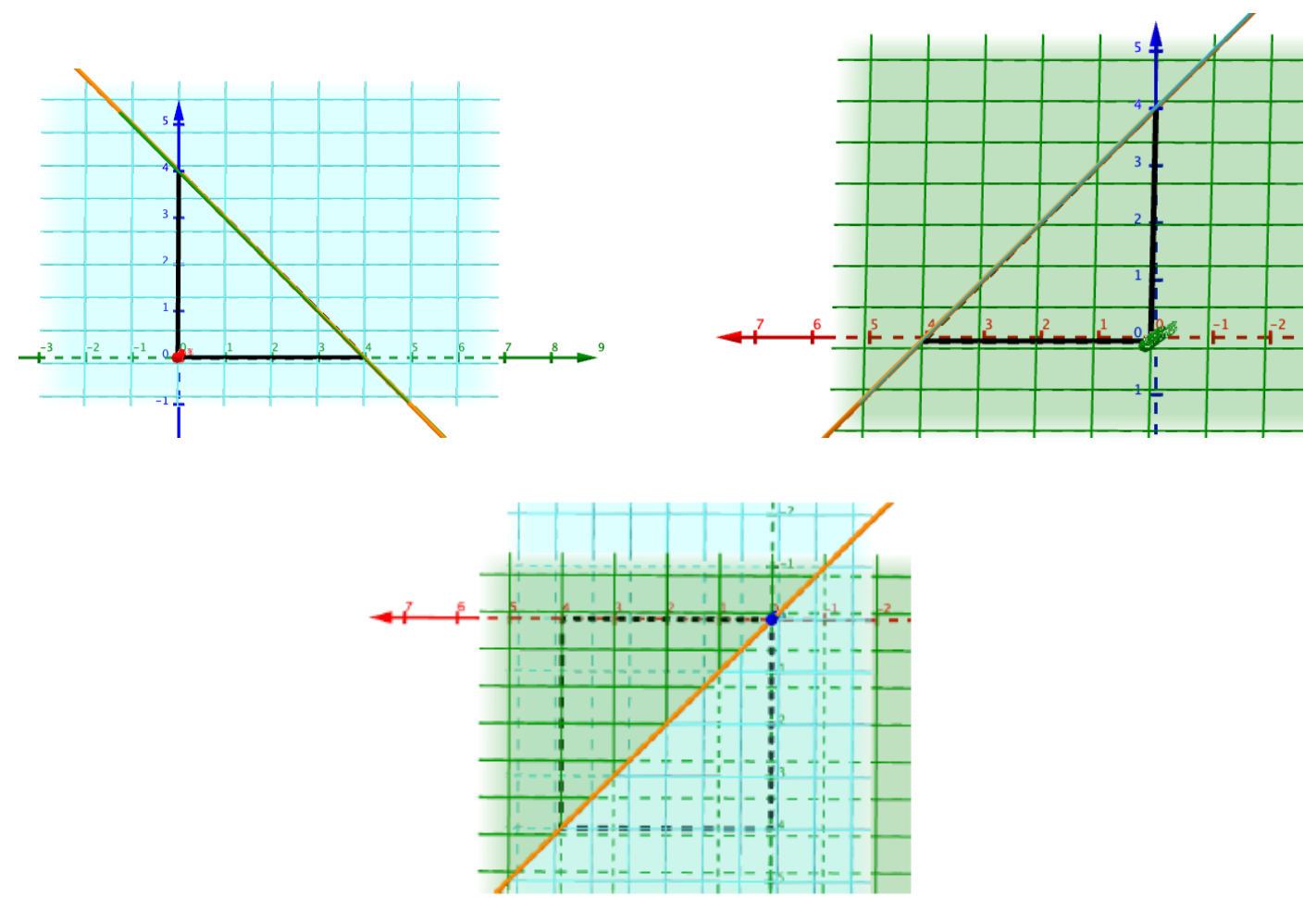

Figura 1.5: Rotación del sólido para observar las proyecciones hecho por una estudiante en GeoGebra 3D.

Los errores cometidos por los educandos fueron mínimos. De hecho, solo uno planteó la integral en el orden $\mathrm{d} z \mathrm{~d} y \mathrm{~d} x$ observando la proyección como el triángulo $\left\{(x, y) \in \mathbb{R}^{2}: 0 \leq x \leq 4,0 \leq y \leq\right.$ x\} y multiplicó la integral triple por dos, lo cual no es del todo erróneo si se estuviera solicitando el volumen de $\mathrm{Q}$. Sin embargo, no se considera como una respuesta completamente correcta.

- Considere el sólido $Q$ limitado por las superficies $z=2 \sqrt{x^{2}+y^{2}} y z=3-x^{2}-y^{2}$.

a. Use una integral triple en coordenadas cilíndricas para determinar el volumen de Q.

b. Plantee una integral triple en coordenadas esféricas que permita determinar el volumen de Q.

Los estudiantes usaron GeoGebra en esta pregunta para visualizar el sólido y la proyección de este sobre el plano $x y$, así como el valor de $z$ en la intersección de las dos superficies dadas, un ejemplo de ello se aprecia en la figura 1.6, el cual fue hecho por un alumno.
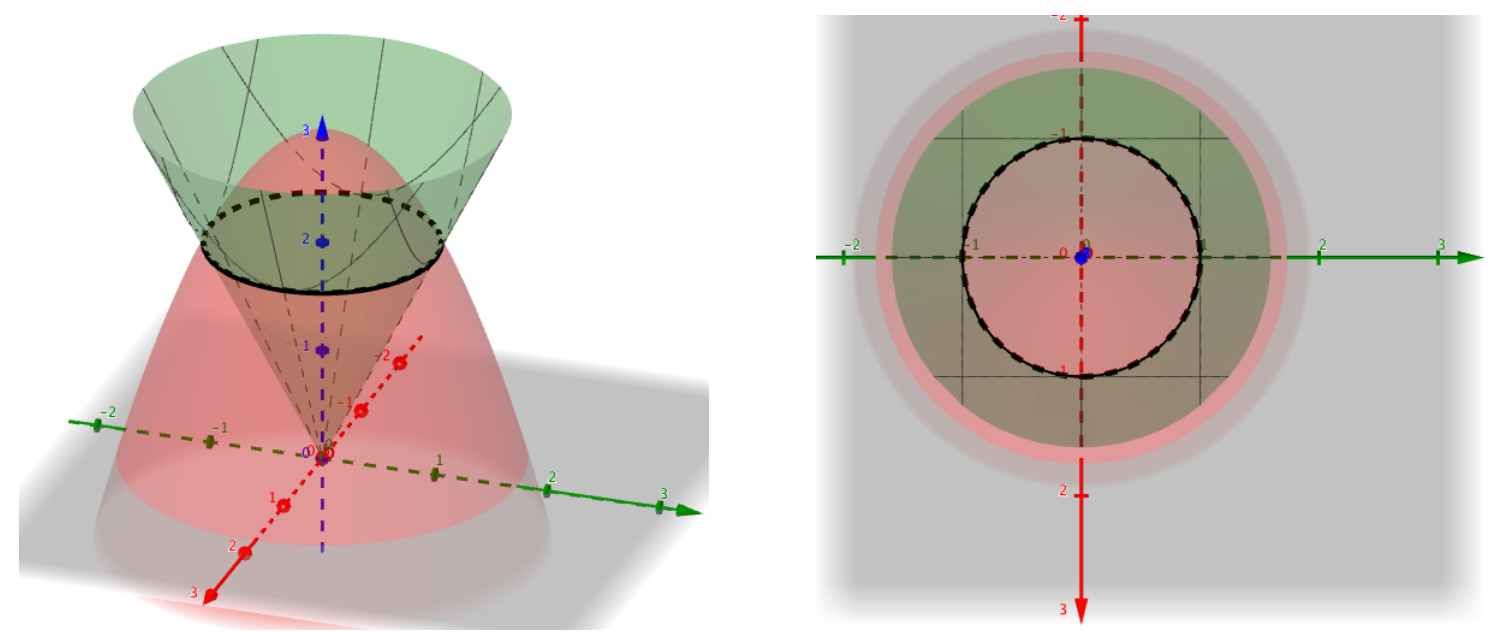

Figura 1.6: Sólido Q y su proyección en el plano xy hecho por un alumno en GeoGebra 3D. 
También usaron la herramienta de rotación para poder visualizar el movimiento del ángulo $\phi$ a la hora de plantear la integral triple en coordenadas esféricas. La figura 1.6 muestra lo que hizo el mismo alumno de la figura anterior.

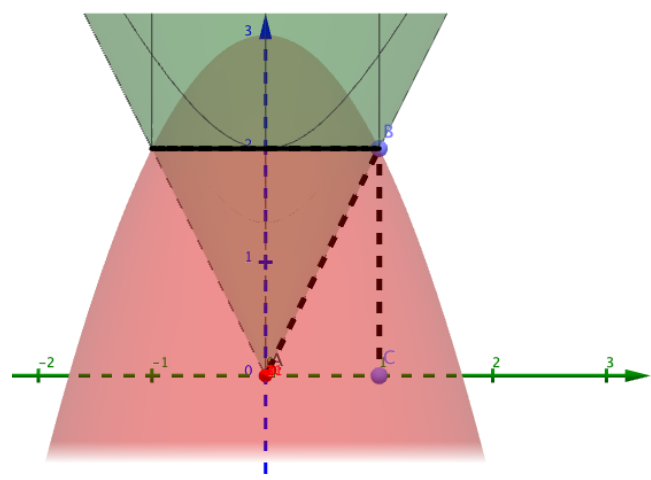

Figura 1.7: Rotación de $Q$ hecho por un alumno en GoeGebra para determinar el movimiento de $\phi$.

Es evidente que todas las vistas anteriores del mismo sólido Q ayudaron a plantear las integrales que se pidieron. Inclusive, algunos educandos al visualizar que dicho sólido es simétrico en cada octante para $z \geq 0$, solo trabajaron con el primer octante y multiplicaron por cuatro la integral triple.

- Sea Q el sólido limitado inferiormente por el paraboloide $a z=x^{2}+y^{2}$ y superiormente por la esfera $x^{2}+y^{2}+z^{2}=2 a^{2}$, con $a \in \mathbb{R}^{+}$.

a. Dibuje el sólido Q.

b. Plantee la integral triple $I=\iiint_{Q}\left(x^{2}+y^{2}+z^{2}\right) d V$ usando coordenadas esféricas en el orden $d \phi d \rho d \theta$.

Esta pregunta plantea una variante en comparación con las demás, que es el uso de un parámetro. En la resolución, lo que hicieron la mayoría de estudiantes era asignarle un valor adecuado al parámetro a para poder dibujarlo en GeoGebra. Por otro lado, únicamente dos estudiantes usaron la herramienta de deslizador en GeoGebra para cambiar el valor de a y visualizar el comportamiento del sólido formado por las dos superficies, según se aprecia en la figura 1.6.

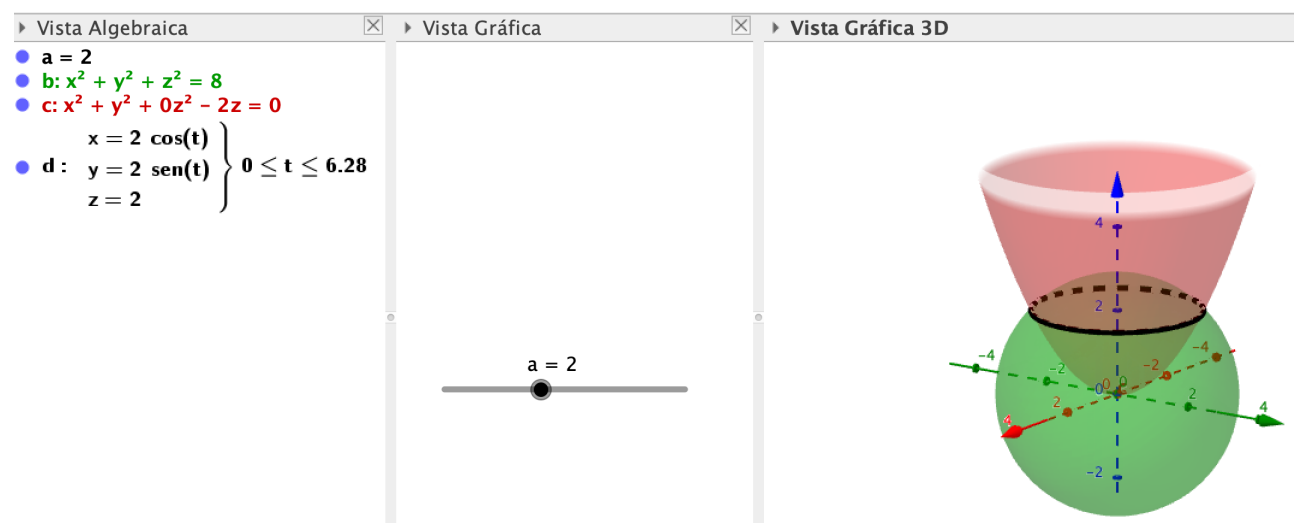

Figura 1.8: Sólido Q con el deslizador del parámetro a hecho por un estudiante en GeoGebra.

Además, la dificultad de esta pregunta también radica en que se solicitó la integral triple en coordenadas esféricas en un orden de integración no usual, para lo cual los estudiantes debieron 
observar detalladamente los intervalos de movimiento del ángulo $\phi$ y del radio $\rho$. Indudablemente, y de acuerdo con las diversas imágenes que aportaron los discentes sobre este ejercicio y las respuestas hasta este punto, al usar la rotación en GeoGebra en tres dimensiones pudieron observar y determinar estos intervalos. No obstante, hubo equivocaciones cuando despejaron $\phi$ en términos de $\rho$, y a partir de ello se arrastra un error grave, consecuentemente no tenían por completo las resoluciones correctas. En fin, solo un estudiante obtuvo bien toda la respuesta.

En resumen, de los 70 puntos del segundo parcial donde se puede recurrir a GeoGebra para indagar sobre las respuestas, la suma promedio obtenida por los discentes fue de 51 puntos. Es decir, un $74 \%$ de aprobación, el cual sigue siendo un porcentaje satisfactorio.

Análisis de los resultados de la tercera prueba parcial. La tercera prueba parcial tenía cuatro preguntas con un valor total de 80 puntos, de los cuales, 60 puntos correspondían al planteo y resolución de integrales de línea e integrales de superficie, incluyendo el Teorema de Green, el Teorema de Stokes y el Teorema de la Divergencia de Gauss, donde los educandos podían apoyarse en GeoGebra para interpretar las posibles resoluciones. Los otros 20 puntos fueron preguntas más formales sobre los temas evaluados, donde el uso del software es de poca o nula utilidad. A continuación se presentan algunas de las interrogantes.

- Considere la curva $x^{3}+y^{3}=3 x y$, llamada el folium de Descartes.

a. Realice un dibujo de dicha curva y coloree la región que encierra en el primer cuadrante. Llame dicha región $R$.

b. Verifique que una parametrización de dicha curva es $r(t)=\left(\frac{3 t}{t^{3}+1}, \frac{3 t^{2}}{t^{3}+1}\right), t \in \mathbb{R}-\{-1\}$.

c. Verifique que $x d y-y d x=\frac{9 t^{2}}{\left(t^{3}+1\right)^{2}} d t$.

d. Use el Teorema de Green para mostrar que el área de R es $\frac{3}{2}$. Sugerencia: note que $0<t<$
$+\infty$.

Cabe destacar que el folium de Descartes es una curva que no se estudió en clases ni en las prácticas. Eventualmente, al ser una curva de la cual posiblemente los estudiantes desconocían sus propiedades y su gráfica, su construcción solo con hoja y lápiz, podía tener una dificultad mayor. Aquí es donde el uso de GeoGebra aporta un gran beneficio, ya que rápidamente se obtienen la curva. De hecho, todos los educandos la dibujaron de forma correcta e identificaron la región R.

Para verificar la parametrización de la curva los estudiantes tenían varias alternativas. Las dos utilizadas fueron: sustituir los parámetros en el folium de Descartes y asegurarse que la igualdad se cumple, y la otra consistió en aplicar la herramienta de trazar curvas parametrizadas en GeoGebra y verificar que realmente coincide, como lo muestra la figura 1.6.

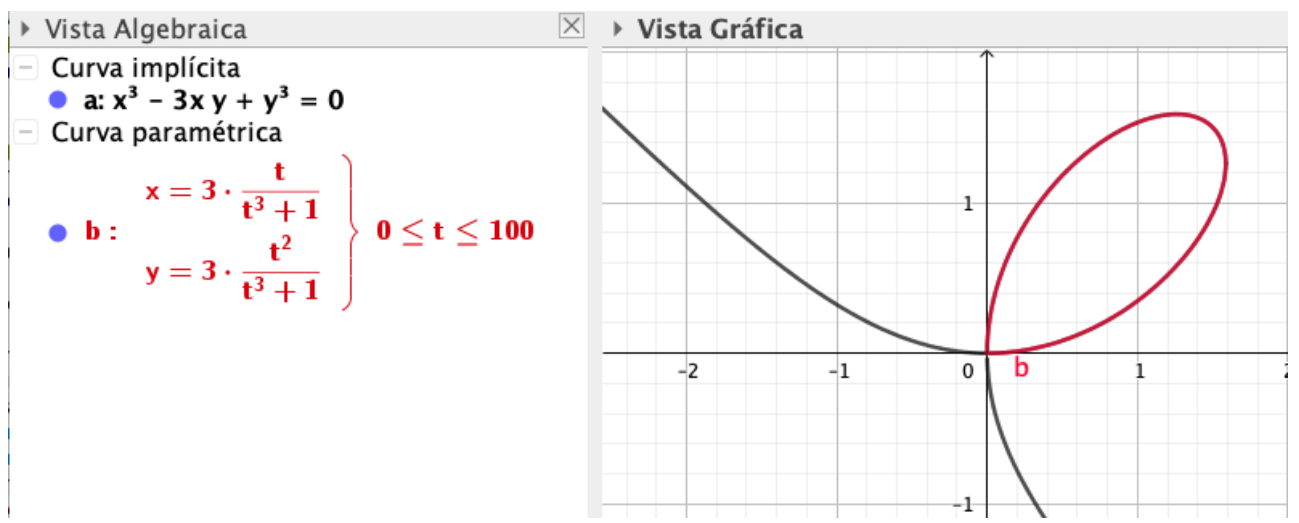

Figura 1.9: Construcción de la curva folium de Descartes hecha por un estudiante en GeoGebra. 
- Considere el sólido cerrado $Q$ acotado por $x^{2}+y^{2}=1, x-z=1$ y $x+z=1$.

a. Realice un dibujo del sólido Q e indique la cantidad de superficies que componen este

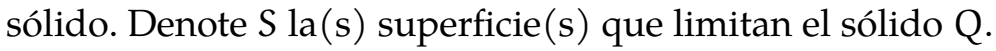

b. Sea el campo vectorial $F(x, y, z)=\left(x y^{2}+2, x^{2} y-2, x^{2} y^{2}\right)$. Muestre que

$$
\iint_{S} \mathrm{~F} \cdot \mathrm{d} S=\pi
$$

usando Teorema de la Divergencia y sin usar el Teorema de la Divergencia.

Aquí GeoGebra en tres dimensiones y la herramienta de rotación, colaboró en gran medida con la resolución pues lo estudiantes pudieron verificar que la frontera del sólido cerrado Q se compone de tres superficies y para definir la dirección del vector normal $\mathfrak{n}$ cuando debían verificar la integral de superficie sin el Teorema de la Divergencia. La figura 1.6 presenta el sólido Q hecho por una discente.

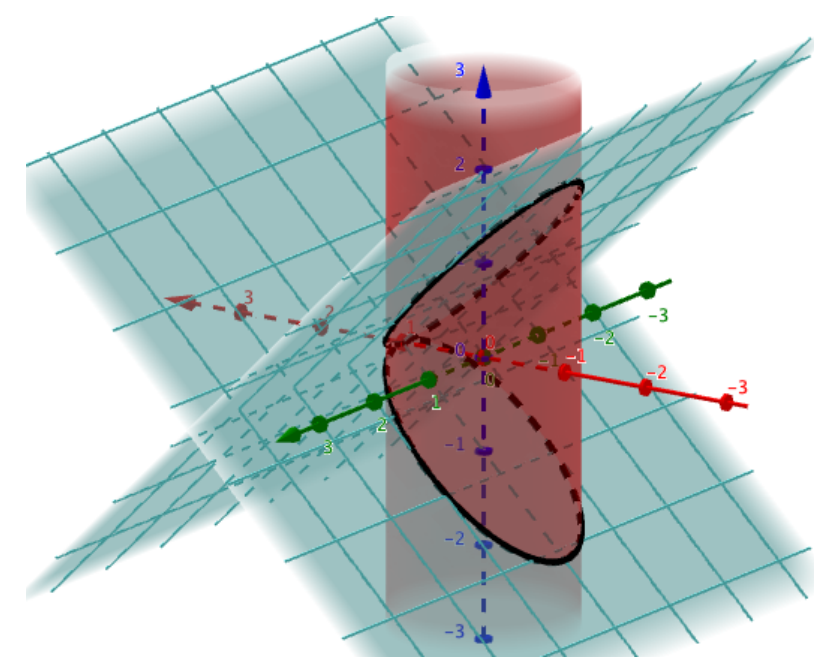

Figura 1.10: Sólido Q compuesto por tres superficies elaborado por una alumna en GeoGebra.

Posteriormente, ya con el detalle del sólido Q obtenido en GeoGebra, los educandos continuaron con la resolución. Se recalca que todos los estudiantes tuvieron bien la dirección del vector $\mathbf{n}$, demostrando el beneficio del uso del software. Sin embargo, los errores más comunes fueron cuando verificaron la integral de flujo sin el Teorema de la Divergencia, y esto en gran medida por errores de cálculo.

- Sea $S$ es la porción del paraboloide $z=x^{2}+\frac{y^{2}}{4}$ que queda por debajo del plano $z=2 x$.

a. Realice un dibujo de la superficie $S$.

b. Aplique el Teorema de Stokes para calcular $I=\iint_{S} \operatorname{rot} F \cdot \mathfrak{n} \mathrm{d} S$, donde $\boldsymbol{n}$ es el vector normal unitario a la superficie $S$ cuya tercera componente es negativa y $F(x, y, z)=(y, z, x)$.

Similarmente a la pregunta anterior, GeoGebra 3D colaboró a una correcta visualización de la superficie formada y su borde, con la cual deben trabajar. Además, esta interrogante tiene la particularidad que solicita el cálculo de una integral de flujo por medio de una integral de línea, por tanto los estudiantes debían parametrizar el borde de la superficie $S$ y la podían verificar gráficamente en GeoGebra. En efecto, la gran mayoría de estudiantes lo hicieron de esa forma. Un ejemplo de ello lo muestra la figura 1.6, donde se observa la superficie $S$ y además la curva paramétrica que corresponde al borde. Los errores cometidos obedecen al mal cálculo de la integral de línea. 


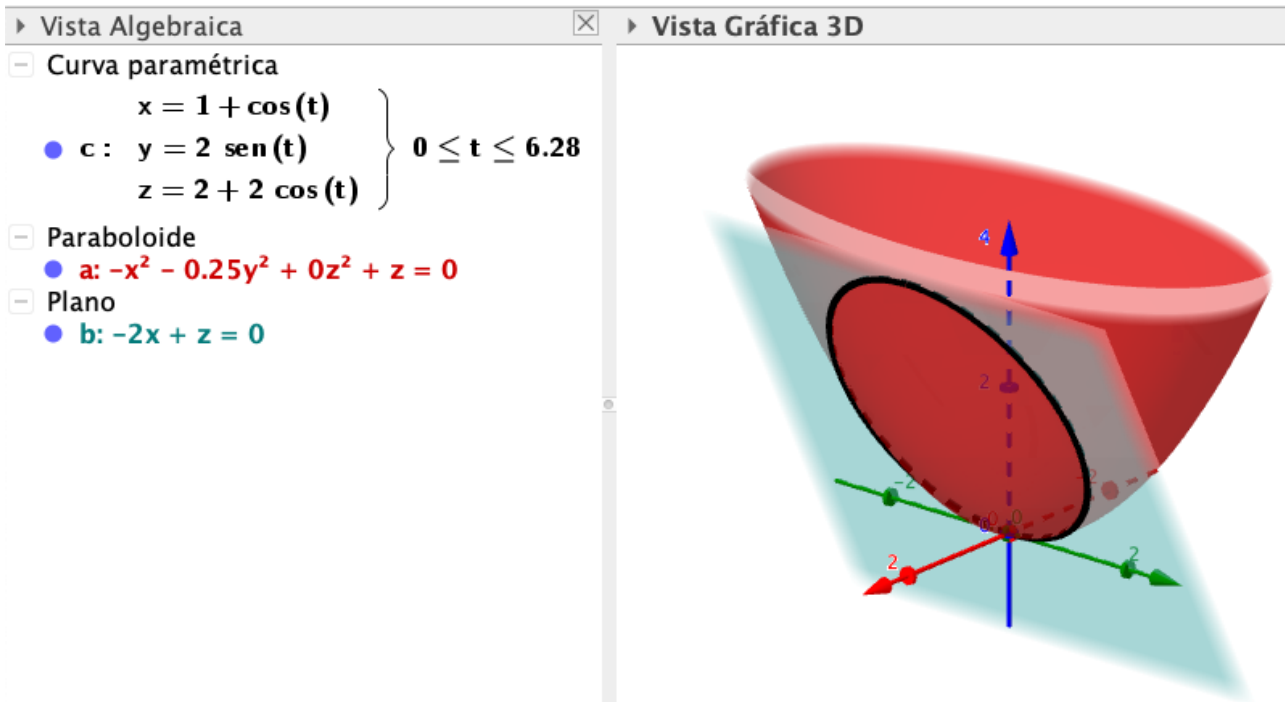

Figura 1.11: Superficie $S$ con su borde parametrizado hecho en GeoGebra 3D por un estudiante.

En síntesis, de los 60 puntos donde era viable usar GeoGebra para poder dar respuesta a las preguntas planteadas, los estudiantes obtuvieron 45 puntos en promedio, para un porcentaje de rendimiento de un $75 \%$, el cual sigue siendo bueno.

Consideraciones finales de la aplicación de las tres pruebas parciales. En relación con todos los resultados anteriores, se deduce que hubo un 75,1\% de aprobación en los tres exámenes parciales en aquellas interrogantes donde era posible emplear GeoGebra para interpretar su solución. Ahora, teniendo presente que usualmente se necesita un $70 \%$ para aprobar un curso universitario, se intuye que el porcentaje obtenido fue satisfactorio.

Por otro lado, se evidencia que GeoGebra en dos y tres dimensiones es de gran ayuda para visualizar curvas y superficies, sobre todo cuando éstas se integran para formar sólidos. Asimismo es una herramienta que permite verificar gráficamente algunos procesos que se están haciendo, como una correcta parametrización de una curva que es la intersección de dos superficies, la dirección de una curva paramétrica, o bien la dirección del vector normal unitario a una superficie, si aplica, los límites de integración, entre otros. Esto, a su vez, colabora con la seguridad de los estudiantes cuando está resolviendo las preguntas propuestas tanto en los exámenes y como en las prácticas.

Además, se evidenció que los educandos adquirieron una mayor destreza con el uso de GeoGebra en tres dimensiones, pues cada vez mostraron dibujos más elaborados con detalles importantes, para lo cual usaron herramientas de rotación, intersección de superficies, superficies parametrizadas y curvas parametrizadas en el espacio, entre otros. Gracias a ello, les permitía una mejor visualización.

\section{Análisis de los datos obtenidos de la observación participante y el cuestionario}

A continuación se presenta la información más relevante que se generó a partir de las observaciones participantes y de las respuestas obtenidas en el cuestionario.

Se evidenció que la mayoría de los educandos tenían conocimiento básicos de GeoGebra, beneficiando la primera semana de clases donde se hacía una explicación general del uso de este software de Geometría Dinámica, sobretodo para graficar en dos y tres dimensiones, incluyendo curvas y superficies, ya sean parametrizadas o no. Los estudiantes realmente se notaron muy motivados por el uso de una herramienta tecnológica reconocida y que además se use para un curso que ciertamente tiene un alto componente de formalidad matemática.

De hecho, algunos comentarios obtenidos del cuestionario son: "evito errores que podría cometer graficando", "me ahorra tiempo", "GeoGebra en 3D me permite visualizar desde varias posiciones en 
poco tiempo", "permite visualizar los límites de una región en 2D", "ayuda a visualizar las fronteras de regiones en 3D", "coadyuva a que se pueda plantear mejor las integrales", entre otros.

Es relevante destacar el último comentario, porque precisamente con el software se pretende contribuir al planteamiento correcto de integrales en varias variables, siendo así el paquete computacional un medio y no un fin, como se escribió al inicio de este apartado.

De forma similar, los alumnos están de acuerdo en que el uso de GeoGebra en 2D y 3D les ha permitido realizar, de forma más eficiente, ejercicios que involucren el Teorema de Green, el Teorema de la Divergencia y el Teorema de Stokes.

Sumado a todo lo anterior, se señalan los siguientes aspectos generales y positivos, los cuales son comentarios textuales de los estudiantes tomados del cuestionario: "me permite orientarme con respecto a un dibujo", "a mí me ha ayudado a comprender mejor, pues realizar las gráficas en 3D es la parte más difícil del curso", "las proyecciones de los dibujos quedan más claras de observar", "permite una mejor visualización e interpretación de las gráficas" y "facilita la clase tanto para el docente como para el estudiante", entre otros.

De acuerdo con las opiniones anteriores, se puede interpretar que realmente el uso de GeoGebra para graficar en dos y tres dimensiones favorece la resolución de integrales en varias variables, pues, a pesar de que un educando posea habilidades numéricas y algebraicas muy buenas que le permitan resolver integrales en más de una variable, sino no ha hecho una correcta gráfica de la región de integración, es probable que la resolución sea inadecuada ya que se puede cometer errores a la hora de plantearla, y es aquí donde el paquete computacional funciona como una alternativa pedagógica para ayudar al estudiante.

Según la observación participante hecha, los estudiantes mostraron seguridad cuando resolvían ejercicios en clase al contar con GeoGebra, y solían llegar a las respuestas correctas con regularidad, esto repercutía positivamente en la motivación. Inclusive, el ritmo de clase fue normal, en comparación con otras clases donde se imparten la misma teoría.

Es importante mencionar los aspectos por mejorar indicados por los estudiantes, los cuales son: "el curso se podía complementar en un laboratorio de informática para no tener que traer las computadoras portátiles", lo cual es una proposición muy atinada, sin embargo, es difícil conseguir un espacio en los laboratorios. Además, "en ocasiones me confiaba demasiado en el dibujo que realizaba en GeoGebra, y perdía procedimientos". En este sentido, se deduce que algunos educandos usaron el software como un fin y no como un medio porque este no resuelve integrales en dos y tres variables.

Otro aspecto por mejorar fue "en algunas veces no lograba emplear las herramientas de GeoGebra de forma correcta" y "se requiere de mayor explicación para el caso de los estudiantes que hemos usado poco GeoGebra". Aunque se usó la primer semana de clases para explicar el uso de GeoGebra de forma general, pareciera que se necesita más tiempo, sobretodo para aquellos discentes que tienen conocimientos nulos sobre este paquete informático. Así, es conveniente al menos trabajar una clase más sobre el uso de GeoGebra y en aquellas funciones que interesan para el curso. Cabe destacar que eran muy pocos los estudiantes con desconocimiento del uso de GoeGebra, como se mencionó en párrafos anteriores. Además de que conforme avanzaba el curso y se resolvían ejercicios con el apoyo e GeoGebra, los estudiantes adquirían mayores destrezas en el uso del software.

\subsection{Conclusiones}

Las principales conclusiones de este trabajo se puntualizan a continuación:

- La implementación de actividades didácticas que utilizan GeoGebra en dos y tres dimensiones 
favorecen el proceso de la enseñanza y el aprendizaje de temas afines a integración en varias variables. Esto obedece a que se beneficia la motivación y por ende el interés de los estudiantes durante las clases, inclusive puede mejorar la comprensión de forma significativa y consecuentemente optimizar el rendimiento académico.

- El estudio de los temas relacionados con integración múltiple, se prestan para usar algún sofware de Geometría Dinámica como recurso didáctico, repercutiendo positivamente en el proceso de enseñanza-aprendizaje, según y como se demostró en esta investigación y se escribió anteriormente. Sin embargo, de forma habitual, estos temas se enseñan de forma tradicional, solo con pizarra y marcador, dejando aún la necesidad de realizar más esfuerzos para cambiar este tratamiento metodológico.

- El uso de algún sofware de Geometría Dinámica como GeoGebra no resuelve de forma definitiva los problemas de enseñanza-aprendizaje asociados a temas de integración múltiple, sin embargo, es un estimable recurso didáctico que permite a los alumnos mejorar el razonamiento matemático y la capacidad para la resolución de problemas.

- De acuerdo con las necesidades de los educandos, el docente es el responsable de diseñar las situaciones didácticas más convenientes para optimizar el uso de GeoGebra (o cualquier otro paquete computacional) y así aprovechar sus potencialidades. También, los estudiantes son responsables de ser partícipes activos de las actividades planteadas y de indicar al docente cuando una actividad fue o no fue provechosa, esto con el fin de mejorar continua y permanentemente.

- Se debe insistir en que el uso de los paquetes informáticos en la Educación Matemática Superior tienen que ser un medio para impactar positivamente las metodologías usadas en las respectivas clases. En este sentido, las medidas adoptadas por el discurso didáctico, donde se utilice software educativos, deben coadyuvar el razonamiento matemático.

Agradecimientos. Se agradece a los estudiantes del curso Principios de Análisis II de la carrera de Licenciatura y Bachillerato en la Enseñanza de la Matemática, de la Sede Occidente, Universidad de Costa Rica, esto durante el II Ciclo semestral del año 2017.

\section{Bibliografía}

[1] Andrade, M. y Montecino, A. (Marzo del 2011). La problemática de la tridimensionalidad y su representación en el plano. XIII Conferenica Interamericana de Educación Matemática. Conferencia llevada a cabo en el congreso Comité Interamericano de Educación Matemática, Recife, Brasil.

[2] Apostol, T. (2004). Calculus. Volumen II. (2da. Ed.). Barcelona: Reverté S.A.

[3] Artigue, M. (1995). La enseñanza de los principios del cálculo: problemas epistemológicos, cognitivos y didácticos. En Artigue, M., Douady, R., Moreno, L., Gómez, P. (eds.), Ingeniería didáctica en educación matemática. México: Grupo Editorial Iberoamérica, 97140.

[4] Barrantes, R. (2014). Investigación: un camino al conocimiento. Un enfoque cualitativo, cuantitativo y mixto. (2da. Ed.). San José: EUNED.

[5] Bisquerra, R. (2009). Metodología de la Investigación Educativa. (2da. Ed.). Madrid: La Muralla S.A.

[6] Carrillo, A. y Llamas, I. (2009). GeoGebra. Mucho más que geometría dinámica. México D.F.: Alfaomega. 
[7] Costa, V.; Di Domenicantonio, R. y Vacchino, M. (2010). Material educativo digital como recurso didáctico para el aprendizaje del Cálculo Integral y Vectorial. Revista Iberoamericana de Educación Matemática, (21), 173-185.

[8] Del Río, L. (2016). Enseñar y aprender cálculo con ayuda de la vista gráfica 3D de GeoGebra. Revista Digital Matemática, Educación e Internet, 17(1), 1-13. Recuperado de: https://tecdigital.tec.ac.cr/revistamatematica/Secciones/Didactica y_Software/RevistaDigital_DelRio_V17_N1_2016/RevistaDigital_DelRio_V17_n1_ 2016.pdf

[9] Dorko, A. y Weber, E. (2014). Generalising calculus ideas from two dimensions to three: how multivariable calculus students think about domain and range. Research in Mathematics Education, 16(3), 269-287.

[10] Gómez, P (1997). Tecnología y Educación Matemática. Informática Educativa, 10(1), 93111.

[11] Gómez, P. y Delgado, J. (2012). La diferenciabilidad de funciones de varias variables. Una propuesta de tratamiento metodológico. Acta Latinoamericana de Matemática Educativa, 25, 626-636.

[12] Infante, P.; Quintero, H. y Logreira, C. (2010). Integración de la Tecnología en la Educación Matemática. Revista Electrónica de Estudios Telemáticos, 9(1), 33-46. Recuperado de: http: //www. redalyc. org/pdf/784/78415022003.pdf

[13] Mora, W. (2019). Cálculo en Varias variables. Visualización interactiva. (2da. Ed.). vcartago, Costa Rica: Revista Digital Matemática, Educación e Internet. Recuperado de: https: //tecdigital.tec.ac.cr/revistamatematica/Libros/

[14] National Council of Teachers of Mathematics. (2015). De los principios a la acción. Para garantizar el éxito matemático para todos. México D.F.: 3D Editorial.

[15] Parra, O. y Díaz, V. (2014). Didáctica de las Matemáticas y Tecnologías de la Información y la Comunicación. Revista Educación y Desarrollo Social, 8(2) , 60-81.

[16] Pita, C. (1995). Cálculo Vectorial. (1er. Ed.). Juárez: Prentice Hall Hispanoamérica S.A.

[17] Salinas, P.; Alanís, J. y Pulido, R. (2011). Cálculo de una variable. Reconstrucción para el aprendizaje y la enseñanza. Didac, 56-57, 62-69. Recuperado de: http://www. sinewton . org/numeros/numeros/88/Articulos_05.pdf

[18] Santana, N. y Climent, N. (2015). Conocimiento Especializado del Profesor para la utilización de Geogebra en el Aula de Matemáticas. Revista de Didáctica de las Matemáticas, 88, 75-91. Recuperado de: http://www. sinewton.org/numeros/numeros/88/Articulos_ 05. pdf

[19] Stewart, J. (2002). Cálculo Multivariable. (4ta. Ed.). México, D.F.: Thomson Learning.

[20] Trigueros, M. y Martínez-Planell, R. (2010). Geometrical representations in the learning of two-variable functions. Educational Studies in Mathematics, 73(1), 3-19.

[21] Vicente, P.; Trefftz, H. y Restrepo, J. (2006). Estrategias de visualización en el cálculo de varias variables. Revista Educación y Pedagogía, 18(45), 75-91.

[22] Vieira, F. (2012). Discussao do uso do GeoGebra no contexto do Cálculo a varias Varáveis. Revista do Instituo GeoGebra de Sao Paulo, 1(2), 05-19.

[23] Weber, E., y Thompson, P. (2014). Students' images of two-variable functions and their graphs. Educational Studies in Mathematics, 87(1), 67-85. 